\title{
On the Spectrum of the Quantum Pendulum
}

N. Kutz

SFB 288 Preprint No. 101

Diese Arbeit ist mit Unterstuitzung des von der Deutschen Forschungsgemeinschaft getragenen Sonderforschungsbereiches 288 entstanden und als Manuskript vervielfältigt worden.

Berlin, Dezember 1993 
square lattice Hofstadter model where found. Furthermore it would be important to study the connection between the above mentioned algebra, their classical counterparts and the therein underlying hamiltonian structure.

\section{Acknowledgements}

I would like to thank A. Bobenko and U. Pinkall for many important discussions, their encouragemnet and their participation in Sect. 2, L. D. Faddeev for turning my attention onto the algebraic Bethe ansatz of the sine-Gordon model and for pointing out reference [WZ] and finally C. Kreft and R. Seiler for many helpful explanations about the underlying physics of the Hofstadter model.

\section{References}

[FV] L. D. Faddeev, A. Yu. Volkov, "Quantum Inverse Transform Method with Discrete Space-Time", Theor. Math. Phys. 92:2, 207-214 (1992) (in Russian)

[V] A. Yu. Volkov, "The Quantum Volterra Model", Preprint Series in Theorethical Physics, Helsinki University-TFT-92-6 (1992)

[BKP] A. I. Bobenko, N. Kutz, U. Pinkall, "The Quantum Pendulum"

[F] L. D. Faddeev, "The Bethe ansatz" Sfb-Preprint Nr. 70 , Berlin (1993)

[WZ] P.B. Wiegmann, A. V. Zabrodin, Preprint LPTENS-93/34

[IK] A. G. Izergin, V. E. Korepin, Lett. Math. Phys 5, 199 (1981)

[FZ] V. Fateev, A. V. Zamolodchikov, Phys. Lett. A 92, 37 (1982)

[STF] E. K. Sklyanin, L. A. Takhtadjan, L. D. Faddeev, Teor.Mat. Fiz.40, 199-205 (1994)

[TTF] V. O. Tarasov, L. A. Takhtadjan, L. D. Faddeev Teor.Mat. Fiz.57:2,163$181(1983)$

[TF] L. A. Takhtadjan, L. D. Faddeev, Russian Math. Surveys 34:5 11-68 (1979)

[W] S. Wolfram, "Mathematica" Second Edition, Addison-Wesley (1991)

[K] . C. Kreft, Sfb-Preprint Nr. 80, Berlin (1993)

[BKS] J. Bellisard, C. Kreft, R. Seiler, J. Phys. A: Math. Gen. 242329 (1991)

[H] D. Hofstadter, Phys. Rev. B 14 2239-2249 (1976) 


\title{
On the Spectrum of the Quantum Pendulum*
}

\author{
Nadja Kutz \\ Techn. Universität Berlin \\ Sekr. MA 8-5, Str. des 17. Juni 136 \\ 10623 Berlin, Germany
}

3.12 .1993

\begin{abstract}
The spectrum of the Quantum pendulum with the anisotropy parameter being a root of unity is expressed in terms of an algebraic Bethe ansatz. Within this context a short survey about the connections between the involved algebras of the sine-Gordon and Volterra model is given.
\end{abstract}

\section{Introduction}

The content of this paper is mainly based on the work of Faddeev and Volkov in $[\mathrm{FV}],[\mathrm{F}],[\mathrm{V}]$. In $[\mathrm{V}]$ the socalled quantum Volterra model was established. This involved in particular the derivation of an quantum Volterra-L-Operator, which satisfies the usual Quantum-Yang-Baxter equation with the trigonometric R-Matrix of the sine-Gordon model. A fundamental L-Operator acting as an R-Matrix on quantum space was constructed. In [FV] it was shown how this integrability could be written as a Quantum Zero Curvature condition on the lattice and quantum evolution operators were constructed. Among others a connection between the quantum Volterra model and the quantum sine-Gordon model was found in $[\mathrm{FV}]$ via the construction of the sine-Gordon L-operator from the Volterra-L-operator by expressing it in terms of the sine-Gordon $\Pi$ and $\Phi$ fields. In $[F]$ the algebraic Bethe ansatz for this L-operator was given.

The fundamental L-operator of the Volterra model is, as found in [V], dependent on a subalgebra of the original Volterra algebra, which we would like to call the C-Algebra. This subalgebra can be depictured as being an algebra on the faces of the lattice rather then being an algebra on the edges of the lattice, as in the case of the original Volterra algebra. Since the Hamiltonian of the Volterra model is

\footnotetext{
*Supported by the Deutsche Forschungsgemeinschaft, Sonderforschungsbereich 288
} 
derived from this fundamental L-operator it depends also only on this subalgebra. In [BKP] it was shown at the example of the monodromy of four lattice sites (the Quantum pendulum) that the trace of a multiple of the Volterra-L-Operator might also be expressed in terms of this algebra. Furthermore it was shown that the zero curvature equation of the Volterra model can be written in this subalgebra, which gives the quantum discrete sine-Gordon equation.

In this paper it will be shown that the C-Algebra is not only a subalgebra of the Volterra algebra, but also of the $\Pi$ and $\Phi$ sine-Gordon algebra. Since it is only a subalgebra, the algebraic Bethe ansatz constructed in $[\mathrm{F}]$ can not fully be applied. Consequently only parts of the spectrum of the Quantum pendula can be recovered in terms of the algebraic Bethe ansatz of $[\mathrm{F}]$.

\section{Connections between the models}

Let us consider the L-operator of the quantum Volterra model [V].

$$
V_{n}(\lambda)=\left(\begin{array}{cc}
u_{n} & -e^{\lambda} v_{n}^{-1} \\
e^{\lambda} v_{n} & u_{n}^{-1}
\end{array}\right)
$$

with $u, v$ obeying the ultralocal commutation relations:

$$
u_{n} v_{n}=e^{-\alpha} v_{n} u_{n} \quad\left[u_{j}, u_{k}\right]=\left[v_{j}, v_{k}\right]=\left[u_{j}, v_{k}\right]=0, \text { if } j \neq k .
$$

It satisfies the fundamental commutation relations (FCR):

$$
R(\lambda-\mu) V_{n}(\lambda, a) \otimes V_{n}(\mu, a)=V_{n}(\mu, a) \otimes V_{n}(\lambda, a) R(\lambda-\mu)
$$

where $R(\lambda)$ is the R-matrix of the Sine-Gordon model (see e.g. [IK],[STF]):

$$
R(\lambda)=\left(\begin{array}{cccc}
1 & 0 & 0 & 0 \\
0 & b(\lambda) & c(\lambda) & 0 \\
0 & c(\lambda) & b(\lambda) & 0 \\
0 & 0 & 0 & 1
\end{array}\right) \text { and } \begin{aligned}
& b(\lambda)=\frac{e^{-\alpha}-e^{\alpha}}{c(\lambda)=\frac{e^{\mu-\alpha}-e^{\lambda-\mu+\alpha}}{e^{\mu-\lambda-\alpha}-e^{\lambda-\mu+\alpha}}} \\
& e^{\lambda-\lambda}
\end{aligned}
$$

The fundamental L-operator $\mathbf{L}_{\alpha, \beta}(\lambda)=\mathbf{P}_{\alpha, \beta} l\left(\lambda, u_{\alpha}^{-1} u_{\beta}^{-1} v_{\alpha}^{-1} v_{\beta}\right)$ satisfies the equation $[\mathrm{V}]$ :

$$
\mathbf{L}_{\alpha, \beta}(\lambda) V_{\alpha}(\lambda+\mu) V_{\beta}(\mu)=V_{\beta}(\mu) V_{\alpha}(\lambda+\mu) \mathbf{L}_{\alpha, \beta}(\lambda)
$$

where $\mathbf{L}_{\alpha, \beta}(0)=\mathbf{P}_{\alpha, \beta}$, is the permutation operator on the two quantum spaces $\alpha$ and $\beta$ and $l$ a function given in [V]. Faddeev and Volkov constructed in [FV] shift operators $U_{ \pm}$which determine the evolution in the light cone directions:

$$
\tilde{V}_{\alpha}(\lambda-\omega)=U_{+}^{-1} V_{\alpha}(\lambda-\omega) U_{+} \quad \tilde{V}_{\beta}(\lambda+\omega)=U_{-} V_{\beta}(\lambda+\omega) U_{-}^{-1} .
$$

Due to (2.3) and (2.2) the zero curvature condition is satisfied:

$$
V_{\alpha}(\lambda-\omega) V_{\beta}(\lambda+\omega)=\tilde{V}_{\beta}(\lambda+\omega) \tilde{V}_{\alpha}(\lambda-\omega)
$$


If one multiplies the Volterra-L-operator on two consecutive lattice sites as in $[\mathrm{FV}]:$

$$
L_{n}(\lambda)=V_{2 n}(\lambda-\omega) V_{2 n-1}(\lambda+\omega)
$$

and defines on each Cauchy surface

$$
\begin{aligned}
& e^{i \Pi_{n}}:=u_{2 n} v_{2 n-1}^{-1} \\
& e^{i \Phi_{n}}:=u_{2 n}^{-1} u_{2 n-1}^{-1} \\
& e_{n}^{a}:=u_{2 n} v_{2 n}^{-1} u_{2 n-1} v_{2 n-1}
\end{aligned}
$$

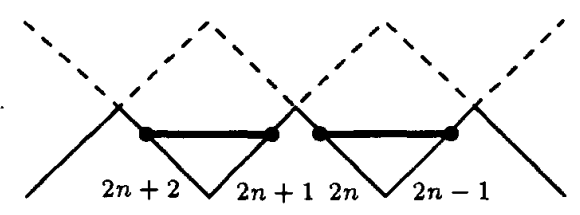

then one obtains the L-operator

$$
L_{n}(\lambda, a)=\left(\begin{array}{cc}
e^{-i \Phi_{n}}-e^{2 \lambda} e^{i \Phi_{n}+a} & -e_{n}^{i \Pi} h_{+}\left(\Phi_{n}, a\right) \\
h_{-}\left(\Phi_{n}, a\right) e^{-i \Pi_{n}} & e^{i \Phi_{n}}-e^{2 \lambda} e^{-i \Phi_{n}-a}
\end{array}\right)=\left(\begin{array}{cc}
L_{n}^{11} & L_{n}^{12} \\
L_{n}^{21} & L_{n}^{22}
\end{array}\right)(\lambda, a)
$$

with

$$
\begin{aligned}
& h_{+}\left(\Phi_{n}, a\right)=e^{\lambda+\omega}+e^{\lambda-\omega} e^{2 i \Phi_{n}-\alpha+a} \\
& h_{-}\left(\Phi_{n}, a\right)=e^{\lambda+\omega}+e^{\lambda-\omega} e^{-2 i \Phi_{n}+\alpha-a}, \quad \text { where } a \in \mathbb{R} ; \lambda \in \mathbb{C}
\end{aligned}
$$

$e^{a}$ is a Casimir in the algebra generated by the $\Pi$ 's and $\Phi$ 's. In [F] it was set equal to one: $e^{a}=1$, but it will be shown in the following that this constraint has to be given up in order to construct the algebraic Bethe ansatz for the Quantum pendulum. $e^{i \Pi}$ and $e^{i \Phi}$ commute again ultralocally, i.e. $e^{i \Pi_{n}} e^{i \Phi_{n}}=e^{\alpha} e^{i \Phi_{n}} e^{i \Pi_{n}}$. Let us define:

$$
C_{n}:=u_{2 n}^{-1} u_{2 n-1}^{-1} v_{2 n}^{-1} v_{2 n-1} \text { and }\left[C_{n}, C_{n-1}\right]=0
$$

Note that this operator already appeared in the fundamental L-operator of the Volterra model (2.3). It may be depicted as being an operator on the faces of the lattice, rather then the $u$ and $v$ which can be looked at as operators on its edges. In [BKP] it was shown that the zero curvature condition of the Volterra model can be written in terms of the above algebra:

$$
C_{u}=\frac{k+e^{\alpha} C_{l}}{1+k e^{\alpha} C_{l}} C_{d} \frac{1+k e^{\alpha} C_{r}}{k+e^{\alpha} C_{r}}
$$

The $C_{i}$ commute nontrivially on adjacent faces only:

$$
\begin{aligned}
& C_{u} C_{l}=e^{2 \alpha} C_{l} C_{u}, \\
& C_{d} C_{l}=e^{2 \alpha} C_{l} C_{u} .
\end{aligned}
$$

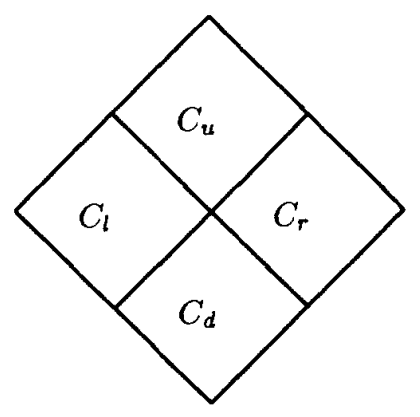


Proposition 2.1 Let $N$ be the period of the lattice, then the product $C_{d_{1}} \ldots C_{d_{N}}$ is preserved by the evolution, i.e

$$
C_{u_{1}} \ldots C_{u_{N}}=C_{d_{1}} \ldots C_{d_{N}}
$$

Proof:

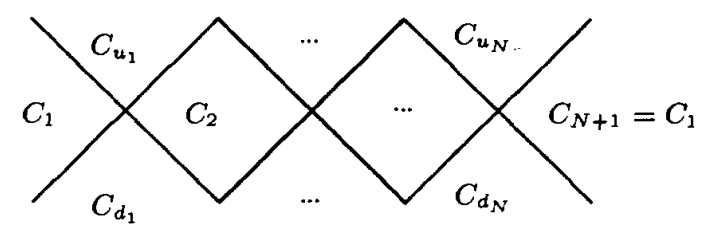

$$
C_{u_{1} \ldots C_{u_{N}}}=\frac{k+e^{\alpha} C_{1}}{1+k e^{\alpha} C_{1}} C_{d_{1}} \ldots C_{d_{N}} \frac{1+k e^{\alpha} C_{N+1}}{k+e^{\alpha} C_{N+1}}=C_{d_{1}} \ldots C_{d_{N}}
$$

since $C_{1}\left(C_{d_{1}} \ldots C_{d_{N}}\right)=\left(C_{d_{1}} \ldots C_{d_{N}}\right) C_{1}$, i.e $C_{d_{1}} \ldots C_{d_{N}}$ is a casimir in the algebra. If $C_{r}$ and $C_{l}$ shall commute in the same way we have to make the following redefinitions :

$$
C_{l}=Q_{l}, \quad C_{r}=Q_{r}^{*}, \quad C_{d}=Q_{d}^{*}, \quad C_{u}=Q_{u}
$$

and for the sake of selfconsistency

$$
C_{l}=Q_{l}^{*}, \quad C_{r}=Q_{r}, \quad C_{d}=Q_{d}, \quad C_{u}=Q_{u}^{*}
$$

Both definitions lead to the same equation $[\mathrm{BKP}]$ :

$$
Q_{u} Q_{d}=\frac{k+e^{\alpha} Q_{l}}{1+e^{\alpha} k Q_{l}} \frac{k+e^{\alpha} Q_{r}}{1+e^{\alpha} k Q_{r}}
$$

which is called the quantum discrete sine-Gordon equation.

Let us consider now the simplest possible case, namely a lattice with faceperiodicity $N=2$.

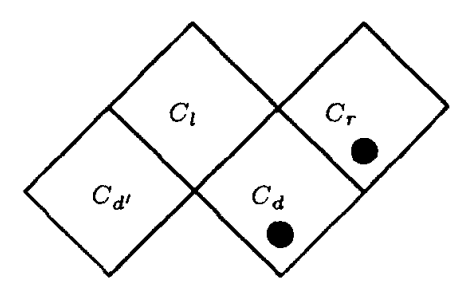

The trace of the monodromy in terms of the C-Agebra reads in this case as:

$$
M(\lambda)=V_{4}(\lambda) \cdot V_{3}(\lambda) \cdot V_{2}(\lambda) \cdot V_{1}(\lambda)=\left(\begin{array}{cc}
A(\lambda) & B(\lambda) \\
C(\lambda) & D(\lambda)
\end{array}\right)
$$




$$
\begin{aligned}
\operatorname{trM}(\lambda)= & A(\lambda)+D(\lambda) \\
= & e^{-\frac{i \beta}{2}-\frac{i \delta}{2}}+e^{4 \lambda} e^{\frac{i \beta}{2}-\frac{i \delta}{2}} \\
& -e^{2 \lambda}\left(e^{-i \beta / 2-i \delta / 2}\left(C_{r}+C_{l}+C_{d}+e^{i \delta} C_{d}+k e^{\alpha} C_{d} C_{r}+\frac{1}{k} e^{\alpha} C_{l} C_{d}\right)\right. \\
& + \text { hermitian conjugate }
\end{aligned}
$$

There are two casimirs:

$$
C_{d} C_{d^{\prime}}=: e^{i \beta} \quad \text { and } C_{r} C_{l}=: e^{i \delta}
$$

Since $Q_{l}$ and $Q_{r}$ obey the same commutation relations with their neighbours, one can set $Q_{l}=Q_{r}$, which refers to the case of a pendulum [BKP]. Taking the above casimirs into consideration we define:

$$
\begin{aligned}
& C_{l}=: Q_{n} e^{i \delta / 2} \Rightarrow C_{r}=: \quad Q^{*} e^{i \delta / 2} \\
& C_{d}=: Q_{n-1}^{*} e^{i \beta / 2} \quad C_{d^{\prime}}=: Q_{n-1} e^{i \beta / 2},
\end{aligned}
$$

where $n$ and $n-1$ refer to the evolution in vertical direction (time).

Proposition 2.2 The trace of the monodromy of (2.10) can be written as

$$
\begin{aligned}
\operatorname{tr} M(\lambda) & =e^{-i \beta}+e^{4 \lambda}-e^{2 \lambda} H(\beta) \\
H(\beta) & =2 \cos \frac{\beta}{2}\left(Q_{n}+Q_{n}^{*}+Q_{n-1}+Q_{n-1}^{*}\right) \\
& +k\left(q^{-1} Q_{n}^{*} Q_{n-1}^{*}+q Q_{n-1} Q_{n}\right) \\
& +\frac{1}{k}\left(q Q_{n} Q_{n-1}^{*}+q^{-1} Q_{n-1} Q_{n}^{*}\right),
\end{aligned}
$$

$$
\beta \in \mathbb{R}
$$

$H(\beta)$ is called the hamiltonian of the Quantum pendulum.

\section{Proof:}

There is a different possibility to define the $Q$-Algebra:

$$
\begin{array}{llll}
C_{r}=: & Q_{n} e^{i \delta / 2}, & C_{d}=: & Q_{n-1} e^{i \beta / 2}, \\
C_{l}=: & Q_{n}^{*} e^{i \delta / 2} & C_{d^{\prime}}=: & Q_{n-1}^{*} e^{i \beta / 2}
\end{array}
$$

If one substitutes both definitions into (2.11) one sees that the Hamiltonian is only invariant under the time-evolution if one demands that $\alpha=\beta$ and hence gets the above hamiltonian (2.12).

Proposition 2.3 Let $H_{H o f}=T+T^{*}+\frac{1}{k}\left(S+S^{*}\right)$ be the Hamiltonian of the Hofstadter model with $S T=e^{i \gamma} ; \alpha=i \gamma$ Then with the Substitutions:

$$
Q_{n}=e^{i \gamma / 2} T S \quad \text { and } \quad Q_{n-1}=e^{-i \gamma / 2} T S^{*}
$$

one has:

$$
k H_{H o f}^{2}-\left(k+\frac{1}{k}\right)=H(\gamma)
$$

Proof: Direct computation.

So if $\beta=\gamma$ the Quantum pendulum gives the square of the Hofstadter Hamiltonian. Generally the Quantum pendulum is a model for a Nearest Neighbour interaction on a lattice. $[\mathrm{K}]$. 


\section{Algebraic Bethe ansatz}

In the last section we learned about the connection between the sine-Gordon model expressed in terms of the $e^{i \Pi}, e^{i \Phi}$ and $e^{a}$-algebra on one hand and via the $C$-algebra on the other hand. In the first case one can write the spectrum of the trace of the monodromy in terms of algebraic Bethe ansatz equations if $e^{a}=1$ $[\mathrm{F}]$. It is possible to generalize this method if one demands that $e^{\frac{a_{1}+a_{2}}{2}}=q^{\rho}$ is a root of unity with $q^{2 n+1}=1$.

For $e^{\alpha}=q$ with $q^{2 n+1}=1$ the operators $e^{i \Pi}$ and $e^{i \Phi}$ have finite dimensional representations:

$$
e^{i \Pi}=e^{i \phi}\left(\begin{array}{cccccc}
0 & 0 & . & . & 0 & 1 \\
1 & 0 & & . & 0 & 0 \\
0 & 1 & 0 & & & 0 \\
. & & . & . & & . \\
. & & & . & . & . \\
0 & & & 1 & 0
\end{array}\right) \quad e^{i \Phi}=e^{i \theta}\left(\begin{array}{cccccc}
1 & 0 & . & . & . & 0 \\
0 & q & 0 & & & . \\
\cdot & 0 & q^{2} & 0 & & . \\
\cdot & & & \cdot & & . \\
\cdot & & & & . & 0 \\
0 & . & . & . & 0 & q^{n-1}
\end{array}\right)
$$

We find in the representation, where $\phi=\pi$ and $\theta=0$ a vacuum state $\Omega$ for the offdiagonal $C(\lambda, a, b)$ of the monodromy in $(2.10)$.

Let $\left\{e_{i}=(0 \ldots, 1, \ldots 0)^{T}\right\}$ be a standard basis on $\mathbb{C}^{2}$, then one obtaines,

$$
\Omega=\sum_{j} f\left(q^{j}\right) e_{j} \otimes e_{-j-\rho-1} \quad \text { with } \quad f\left(q^{j}\right)=q^{-j^{2}-p j} \mathcal{F}(q^{-1} \underbrace{q^{-2 j-\rho}}_{w})^{-1} .
$$

where $\mathcal{F}$ satisfies the functional equation

$$
\frac{\mathcal{F}\left(q^{-1} w\right)}{\mathcal{F}\left(q^{1} w\right)}=\frac{\tilde{k}+w}{1+\tilde{k} w}, \quad \tilde{k}=q k \quad \text { and } \quad w^{2 n+1}=1, \quad k \neq 1 .
$$

A solution of this equation was proposed in [FZ] and [V] with

$$
\mathcal{F}=\sum_{-n}^{n} l_{j} w^{j} \quad \text { and } \quad l_{j}=\prod_{l=1}^{j} \frac{q^{l-1}-\tilde{k} q^{-l+1}}{q^{-l}-\tilde{k} q^{l}}
$$

Following the method of the algebraic Bethe ansatz we know now that

$$
C(\lambda, \rho) \Omega=0, \quad A(\lambda, \rho)+D(\lambda, \rho) \Omega=\Lambda(\lambda, \rho) \Omega
$$

and

$$
(A(\lambda, \rho)+D(\lambda, \rho)) B\left(\lambda_{1}, \rho\right) B\left(\lambda_{2}, \rho\right) \ldots B\left(\lambda_{k}, \rho\right) \Omega=\Lambda\left(\lambda, \rho, \lambda_{1}, \lambda_{2}, \ldots, \lambda_{k}\right) \Omega
$$

where $\lambda_{1}, \lambda_{2}, \ldots, \lambda_{k}$ have to be solutions to the Bethe ansatz equations $\left(e^{2 \lambda_{i}}=: z_{i}\right)$ :

$$
\prod_{\substack{i \neq j \\ i=0}}^{N-1} \frac{z_{j} q-z_{i} q^{-1}}{z_{j} q^{-1}-z_{i} q}=q^{2 \rho} \frac{q+z_{j}^{2} q^{-1}+z\left(k+\frac{1}{k}\right)}{q^{-1}+z_{j}^{2} q+z\left(k+\frac{1}{k}\right)}
$$

So, in principle, if one would solve the Bethe ansatz equation one could compute the spectrum of $A(\lambda, \rho)+D(\lambda, \rho)=: \operatorname{tr} M(\lambda, \rho)$. 


\section{On the Spectrum of the Quantum Pendulum}

The C-algebra is not only a subalgebra of the $u$ and $v$-algebra, but may be expressed also via the $e^{i \Phi}, e^{i \Pi}, e^{a}$-algebra. In the case of the Quantum pendulum this reads as:

$$
\begin{aligned}
Q_{n} & =e^{i \Pi_{2 n}-i \Pi_{2 n-1}} e^{i \Phi_{2 n}-i \Phi_{2 n-1}-\alpha} \\
Q_{n-1} & =e^{i \Phi_{2 n}-i \Phi_{2 n-1}} \\
e^{i \delta_{n}} & =e^{-\left(a_{2 n}+a_{2 n-1}\right)} \\
e^{i \beta_{n}^{o p}} & =e^{2 i \Phi_{2 n-1}+2 i \Phi_{2 n}+a_{2 n}+a_{2 n-1}}
\end{aligned}
$$

We see that the operator $e^{i \beta}$, which is a casimir in the C-algebra is not anymore a casimir in the algebra of $e^{i \Pi}, e^{i \Phi}, e^{a}$, nevertheless, $e^{i \beta_{n}^{o p}}=e^{2 i \Phi_{2 n-1}+2 i \Phi_{2 n}} q^{2 \rho}$ is a constant of motion and we have:

$$
\begin{aligned}
e^{\frac{i \beta_{1}^{o p}}{2}} \Omega & =e^{i \Phi_{2}+i \Phi_{1}} q^{\rho} \sum_{j=0}^{2 n} f\left(q^{j}\right) e_{j} \otimes e_{-j-\rho-1} \\
& =\sum_{j=0}^{2 n} q^{j} q^{-j-1-\rho} q^{\rho} f\left(q^{j}\right) e_{j} \otimes e_{-j-\rho-1} \\
& =q^{-1} \Omega .
\end{aligned}
$$

Furthermore, it can be shown that with

$$
\phi_{l}\left(\lambda_{1}, \lambda_{2}, \ldots, \lambda_{l}, \rho\right):=B\left(\lambda_{1}, \rho\right) B\left(\lambda_{2}, \rho\right) \ldots B\left(\lambda_{l}, \rho\right) \Omega
$$

one has

$$
e^{\frac{i \beta_{o p}}{2}} \phi_{l}\left(\lambda_{1}, \lambda_{2}, \ldots, \lambda_{l}, \rho\right)=q^{-(l+1)} \phi_{l}\left(\lambda_{1}, \lambda_{2}, \ldots, \lambda_{l}, \rho\right) .
$$

Consequently if we define $e^{\frac{i \beta_{l}}{2}}:=q^{-(l+1)}$ and $k \neq 1$ we know that $\phi_{l+(2 n+1) j}\left(\lambda_{1}, \lambda_{2}, \ldots, \lambda_{l}, \rho\right)$ is a $j$ 'th eigenstate of

$$
H=2 \cos \frac{\beta_{l}}{2}\left(Q_{n}+Q_{n}^{*}+Q_{n-1}+Q_{n-1}^{*}\right)+k\left(q^{-1} Q_{n}^{*} Q_{n-1}^{*}+q Q_{n-1} Q_{n}\right)+\frac{1}{k}\left(q Q_{n} Q_{n-1}^{*}+q^{-1} Q_{n-1} Q_{n}^{*}\right)
$$

so the algebraic Bethe ansatz of the generalized Sine-Gordon model would - if one could solve the Bethe ansatz equations and if these are sufficient - give the spectrum of the Quantum pendulum for all $e^{i \frac{\beta_{l}}{2}}=q^{-(l+1)}$.

Unfortunately e.g. the square lattice Hofstadter hamiltonian doesn't fall into this class, since there $e^{i \beta}=q$. 
In the following figure we see how the eigenvalues of $\Omega$ are distributed within the band spectrum of the Quantumpendulum for $k=2$. On the $y$-axis are the values of $\gamma$ and on the $x$-axis the values of the energy given.

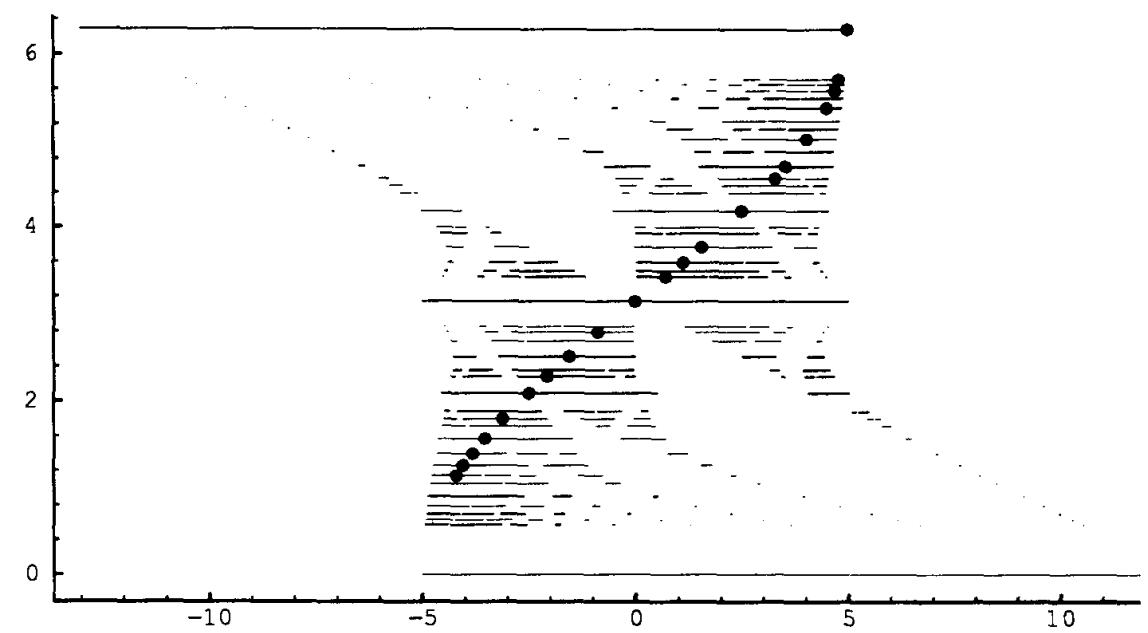

The eigenfunction $\Omega$ in a representation with $N=41$ and $k=2$.

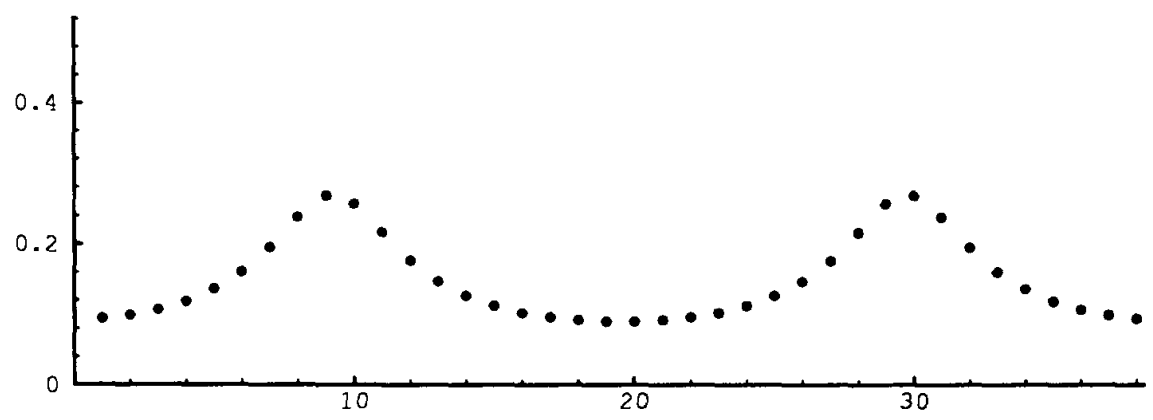

\section{Conclusion}

An interesting approach for further investigations would be the application of the functional Bethe ansatz as e.g. in [WZ], where Bethe ansatz equations for the 